Session 1253

\title{
Teaching Mechatronics To First-Year Engineering Students
}

\author{
Saeed B. Niku \\ California Polytechnic State University, San Luis Obispo
}

\begin{abstract}
:
A number of experiments have been prepared consistent with the knowledge level of freshman engineering students to familiarize them with the concepts of mechatronics. The experiments are based on both dissection of products as well as experimentation with components which comprise mechatronic devices.

Mechatronic devices are thought to be products with mechanical components which perform their function under a microprocessor based controller. Thus, products and components used for this purpose fit the definition.

The experiments are based on a Mini-Board controller, a microprocessor based controller originally designed at MIT and now under production by Progressive Solutions of Columbus, Ohio. The Mini-Board comes equipped with input and output ports, microprocessor, memory, and documentation. It runs programs in Micro-C which are downloaded into it from a computer via the serial port. Lab experiments include connecting the components to the board, development of small programs for controlling d.c. motors and stepper motors, control of lights and buzzers, and conditional control with sensors such as micro-switches, light sensors, and proximity sensors.
\end{abstract}

The dissection modules include investigation of a video camera and its components.

\section{Introduction:}

Mechatronics, as an interdisciplinary subject created by the marriage between mechanical design, microprocessor control, and electronics, has become one of the important new subjects in many institutions. New labs and courses are offered in many universities in order to prepare students with very little background in electronics and micro processors control to design devices which are controlled by a micro processor. There are also many books published about mechatronics which are written specifically for engineers interested in mechatronics ,,,, The trend is partially fueled by the Industry which demands that their new employees be knowledgeable in this area. The result has been course offerings in the senior years in many disciplines.

Additionally, many institutions have been supported by the National Science Foundation, both directly and through NSF Coalitions, to create programs in Mechatronics and to prepare teachers in mechatronics . Additionally, NSF has supported the idea of inclusion of mechatronic concepts in other courses, including first year engineering courses (Synthesis Coalition).

The basic thrust of this project is to introduce students to mechatronics ideas and concepts in their first year engineering course although they do not yet have the basic necessary knowledge. As a result, it is essential that in every project all introductory steps that will be necessary be included, on a need-to-know-basis. 
The experiments in this project are based on a Mini-Board controller, a microprocessor based controller originally designed at MIT and now under production by Progressive Solutions of Columbus, Ohio. The MiniBoard comes equipped with input and output ports, microprocessor, memory, and documentation. It runs programs in Micro-C which are downloaded into it from a computer via the serial port. Students will assemble or connect mechatronic related components to the processor, will develop programs by modifying sample programs, and will test the results. issues.

The following is a description of the micro processor used, experiments, programs, and other related

\section{The Mini-Board:}

The Mini-Board 2.0 is a single-board micro processor designed for running motors and other output devices and for receiving input data from sensors. Its low cost, low power requirements, small size, and programmability make it a useful controller for mechatronics applications. It can communicate with a desk top computer through an RS-232 port. It can work with output voltages of 6 to 36 volts, and through software controlled Pulse Width Modulation, can output 16 different voltage levels in either polarity. It also accepts eight digital and eight analog inputs. Mini-Boards can be networked together and be controlled by a desktop computer, by one of the Mini-Boards, or with distributed control. It also has 256 bytes of internal RAM and $2 \mathrm{~K}$ bytes of EEPROM.

The Mini-Board has four motor ports which can be used for driving output devices such as servo motors, stepper motors, lights, etc. Each port can be specified as either off or on with voltage levels between -16 to +16 (full power at either direction). With proper programming, a stepper motor can also be driven in unipolar, bipolar, or bifilar modes.

There are eight analog input ports on the Board. Each port reports a number from $\mathrm{O}$ to 255 for $\mathrm{O}$ to 5 volts sensed at the port. The input may come from micro switches, proximity switches, light detecting diodes, infra-red detectors, potentiometers, or other such devices.

There are also eight digital input ports on the Board. Each port returns the logical inverse of the actual voltage sensed. They can be used with switches, push buttons, and other digital devices.

In addition, there are special switches, a timer, and a counter port available on the Mini-Board. On of the pins on this port can be used to generate audible sounds with a piezo speaker and be used as a beeper. The above mentioned ports are used for mechatronic experimentation with the first year students.

Although these experiments are based on the Mini-Board, any controller can be used for the same purpose. The only reason for using the Mini-Board is its relatively cheap cost, availability, and reliability.

\section{Experiments:}

A set of simple experiments are developed for the first year engineering students to become familiar with mechatronics concepts. These experiments are designed to be performed in one or two lab periods, and as such, they are short, to the point, and straight forward. They are built on previous experiments and the programming is done mostly by modifying either a blank program or the programs used with previous experiments.

This mechatronics experience is only a part of the Mechanical Systems (ME-134) course at Cal Poly, San Luis Obispo, and not a separate course in itself. As a result, it only takes two weeks to finish them. The rest of the lab time is spent on dissection and design modules, experiments with mechanical items such as springs and bolts, with industrial panels, and CAD. 
The experiments involve the following five parts. For each part, the necessary components are given to the student groups, along with a schematic drawing for connections. Students wire the parts, including the Mini-Board, and check them. The experiment hand out describes the parts, how they work, and what is supposed to happen during the experiment. Along with the schematic, parts, and the description, each part of the experiment has a Micro-C program, called BLANK, which students load and modify according to the instruction. As was mentioned before, since most first year students have little or no experience with mechatronics and $\mathrm{C}$ programming, most basic instructions should be given to them. The BLANK programs provide this easy medium, since students will not have to actually write a program, but modify it or add lines to it. When the program is modified and compiled, it is downloaded to the Mini-Board and is tested according to instructions. When the results are verified and evaluated by both the students and the instructor, students continue with the next part.

Experiment number 1 involves digital input and analog output. A light bulb is turned on and off from the motor port when a switch, connected to the digital input port, is turned off or on.

Experiment number 2 involves digital input and digital output. A proximity sensor connected to the digital input port activates a beeper connected to the special functions port of the Mini-Board. When a magnet is about 1 to $1.5 \mathrm{~cm}$ from the sensor, the beeper will beep.

Experiment number 3 involves analog input and analog output. The speed of a motor connected to the motor port of the Board is controlled by the intensity of the light projected unto a light detector, connected to the analog input port. In response to the intensity of the light, the Mini-Board will turn the motor on and will drive it at different speeds.

Experiment number 4 involves running and controlling a stepper motor. When a digital input from a sensor or switch is received, a stepper motor will be turned on, and it will rotate according to a pre-determined set of motions involving different speeds, different angles, and different directions. Students may change the sequence of motion by editing the program and down-loading it to the Mini-Board. Programming this portion of the experiment is perhaps the most involved, as students will have to create sections for running (angle of rotation), speed control, and direction control of the stepper motor. For more sophisticated students, stepper drivers and indexers may also be added.

Experiment number 5 is a design project, and will be performed with more input from the student group. They will have to consider a function composed of the parts they have used or other components that are similar in nature, connect the parts, modify their program, and run the experiment. A typical set up may be to receive a signal, turn on a device upon receipt of the signal, look for an input from a sensor which may be attached to the motor or may be affected by it, and in turn, turn off the motor when the signal is received. A more sophisticated set up may also include warning lights, variable speeds, beepers, relays, etc. The intention is to force the students to think in terms of mechatronics design and implementation without overwhelming them unnecessarily.

\section{Inclusion of Fuzzy Logic in the experiments:}

It is in fact possible to easily integrate fundamental fuzzy logic control into the experiments as well. Although first year engineering students have no knowledge of control theory, a simplified lecture on fuzzy logic control can prepare them for experimentation with the system. This will certainly add to the number of labs, at least two periods. A medium such as Motorola's FUDGE environment or the Fuzzy Inference Development Environment (FIDE) by Aptronix can be used for this purpose. Experiments on fuzzy logic using FIDE have already been successfully incorporated into Robotics course at Cal Poly, and experience shows a simpler version can be incorporated into the first year engineering course as well. For this purpose too, students may be asked to develop the system on their own by first developing the input and output membership functions and the rule base for controlling a function such as light intensity and motor speed. A blank program which only requires typing the rules and membership functions into the program will enable students to develop and test the fuzzy controller. The developed code can then be similarly downloaded to the Mini-Board and be tested. Although this has not yet been implemented, we plan to incorporate it next year. 


\section{Dissection of a Video Camera:}

So far, a VHS video camera has been dissected for observation by students. At this point it appears that it would not be feasible to have students dissect a camera on their own and re-assemble it back. This is because cameras are very complicated and re-assembling them takes much effort, and that cameras are very expensive. Alternately, the dissected camera will be made available to students for studying, identifying parts, and observation of the relationship between parts, manufacturing, and mechatronics elements. However, in this class students do dissect other devices such as engines, drill motors, and rotary sprinklers.

As an example of an activity on the dissected camera, students are asked to identify parts that are used for a certain function such as auto-focus, determine the function of each part such as the ultrasonic sensor, the micro processor, the motor, the gearbox, and the lens, draw a block diagram of the system, and describe the function of each component. This exercise shows the students what a mechatronic device is and how it is designed.

\section{Sample Blank Programs:}

A sample blank program for Experiment 3 is provided for reference. The program, of course, may be written differently. However, to make it easy for freshman students who may have no knowledge of $\mathrm{C}$ programming, the structure of the program is provided, and minimal editing is required for operation.
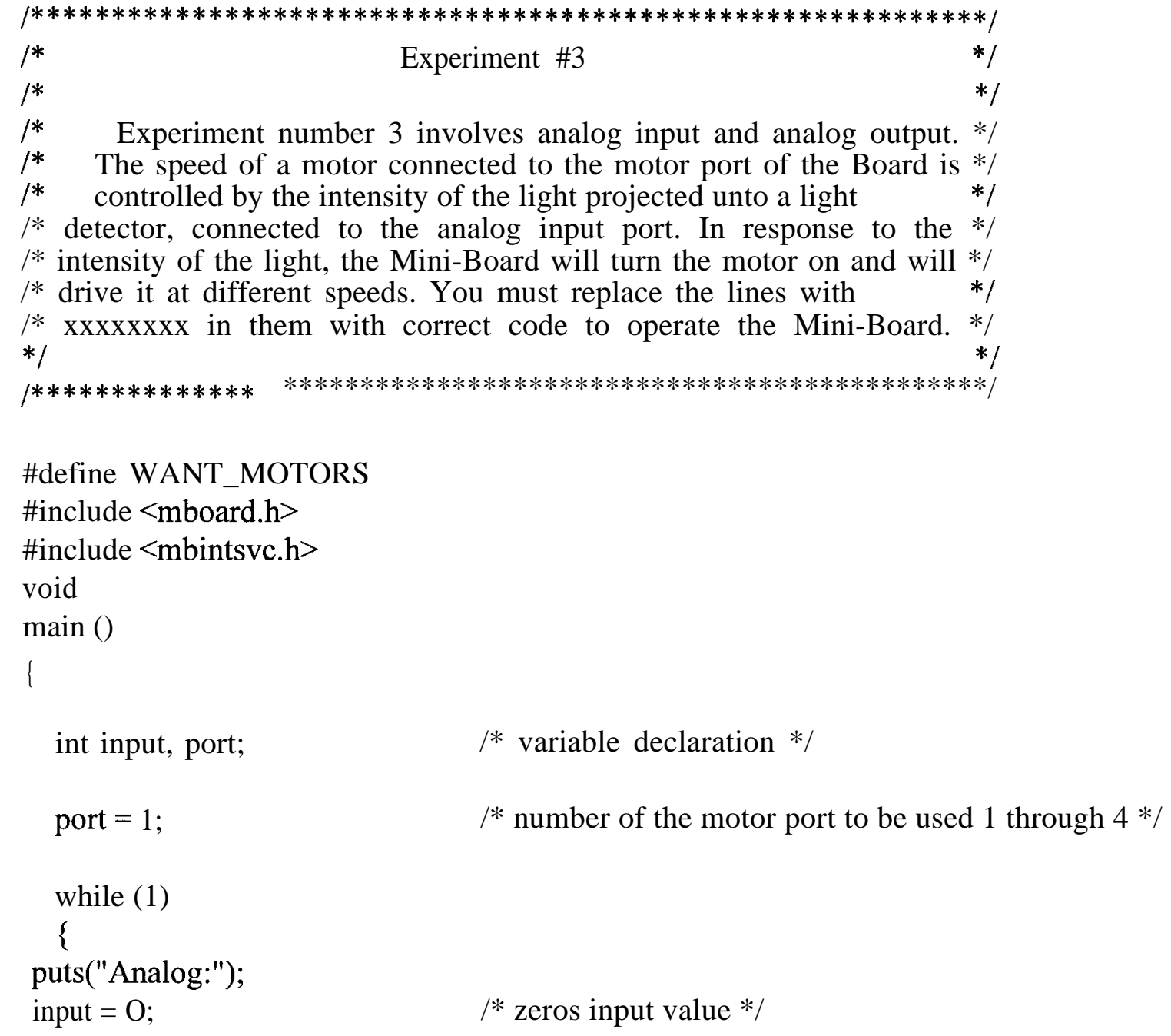

\section{1}


$\operatorname{Xxxxxxxxxxxxxxxxxxx}$

input $=$ input $/ 15$

values $* /$

$\operatorname{XxXXXXXXXXXXXXXXXx}$

\}

\}

In this sample BLANK program, the two lines with $\operatorname{xxxxx}$ in them must be edited by students for proper operation. The substitutes are input = analog (1); and motor (port, input);:

\section{Components Used in the Experiments:}

In addition to the Mini-Boards mentioned above, a collection of stepper motors, D.C. motors, relays, transistors, capacitors, proximity sensors, micro-switches, infrared sensors, buzzers, photo resistors, light bulbs and other miscellaneous parts will be made available to each group of students for experimentation. They may assemble their projects on a proto-board in connection with the Mini-Board.

\section{Acknowledgments:}

The author would like to thank the National Science Foundation's Synthesis Coalition for its support of the project.

\section{References:}

1. Stadler, Wolfram, “Analytical Robotics and Mechatronics”, McGraw Hill, 1995.

2. Stiffler, Kent, "Design with Micro-processors for Mechanical Engineers”, McGraw Hill, 1992.

3. Kamm, L. J., "Understanding Electro mechanical Engineering, An Introduction to Mechatronics", IEEE Publications.

4. Fraser, C., J. Milne, “Electromechanical Engineering, An Integrated Approach’,,IEEE Publications

5. Bergsman, Paul, "Controlling the World with Your PC", HighText Publications Inc.

6. "Preparing The Leaders for Mechatronics Education Workshop", sponsored by NSF, Cal Poly, San Luis Obispo, Ca., June 1995.

7. Martin, Fred, "Mini-Board Technical Reference”, The Progressive Solution, 1992.

\section{SAEED B. NIKU}

Saeed Niku is a professor of Mechanical Engineering at California Polytechnic State University, San Luis Obispo. He received his B. SC. from Tehran Polytechnic University, his M. SC. from Stanford University, and his Ph.D. from the University of California, Davis. He is registered Professional Engineer in State of California. 